University of Wollongong

Research Online

Faculty of Engineering - Papers (Archive)

Faculty of Engineering and Information

Sciences

$1-1-2011$

\title{
Modelling and performance enhancement of a linear actuation mechanism using conducting polymers
}

\author{
Elise T. Burriss \\ etb984@uow.edu.au \\ Gursel Alici \\ University of Wollongong, gursel@uow.edu.au \\ Geoffrey M. Spinks \\ University of Wollongong, gspinks@uow.edu.au \\ Scott McGovern \\ scottmcg@uow.edu.au
}

Follow this and additional works at: https://ro.uow.edu.au/engpapers

Part of the Engineering Commons

https://ro.uow.edu.au/engpapers/1635

\section{Recommended Citation}

Burriss, Elise T.; Alici, Gursel; Spinks, Geoffrey M.; and McGovern, Scott: Modelling and performance enhancement of a linear actuation mechanism using conducting polymers 2011, 63-78.

https://ro.uow.edu.au/engpapers/1635

Research Online is the open access institutional repository for the University of Wollongong. For further information contact the UOW Library: research-pubs@uow.edu.au 


\title{
Modelling and Performance Enhancement of a Linear Actuation Mechanism Using Conducting Polymers
}

\author{
Elise T. Burriss ${ }^{1}$, Gursel Alici ${ }^{2}$, Geoffrey M. Spinks ${ }^{1,2}$, and Scott McGovern ${ }^{1}$ \\ ${ }^{1}$ Intelligent Polymer Research Institute \\ ${ }^{2}$ School of Mechanical, Materials and Mechatronic Engineering \\ University of Wollongong, 2522, NSW, Australia \\ (etb984, gursel,gspinks, scottmg\}@uow.edu.au
}

\begin{abstract}
In this paper, we report on our investigation into modelling and performance enhancement of a linear actuation system based on cantileveredtype conducting polymer actuators, which can operate in air and aqueous media. We have employed the model to predict the linear displacement and force output of the actuation system, and to determine the optimum values of the system design parameters. The linear actuation system is a five bar parallel mechanism, articulated with two polymer actuators. Kinematic and force analyses of the mechanism including numerical results are presented, and its payload handling ability was experimentally evaluated. The experimental results prove that it is possible to generate an accurate linear movement and a corresponding rectilinear force from this mechanism. This mechanism can be employed as a motion and force transmission mechanism, which not only has a light weight, but also consumes a small electrical power.
\end{abstract}

Keywords: Electroactive Polymer Actuators, System Optimization, Linear Actuation, Kinematic Design.

\section{Introduction}

Conducting polymers are favourable as actuators due to their low actuation voltage, high force output relative to their weight, good strain properties, light weight, simple structure and silent motion. The common materials used for actuators are Ionic Polymer Metal Composite (IPMC), and electroactive polymers (EAPs) such as Polypyrrole (PPy) and Polyaniline (PANI) [1]. Disadvantages of polymer actuators include low speed of response, cyclic fatigue and non-linearity, which increases difficulty in modelling and controlling the actuator [2-3].

Bending actuators have been successfully used in applications where a nonlinear motion is required, such as a fin in an artificial fish [4] and robotic fingers [5]. However, the bending motion may limit the control of actuators along a linear path. Conducting polymers have been used as actuators in applications such as medical devices, toys, digital camera accessories and artificial muscles for robots as developed by Eamex [3]. Various PPy linear actuator designs have been investigated, including 
were connected in parallel and immersed in a $\mathrm{LiClO}_{4}$ aqueous solution, were able to produce a displacement of $60 \%$ of the actuator length in the longitudinal direction of the actuator. The use of linear actuators in air may provide an alternative when encapsulation of the electrolyte is not feasible. Yamakita et. Al [7] have developed an IPMC linear actuator for use in a biped walking robot. PPy actuators are used in applications where the oxidised or reduced position is maintained under a constant voltage, for which IPMC is not suitable as it will return to its original position. To overcome this limitation of IPMC, Thompson [1] adopted the design by Yamakita et al. using polypyrrole film as the active layers of a multi-layer actuator structure. Two methods of connecting the PPy film together to produce the linear design were employed by Thompson: (i) masking part of the film during PPy growth to create a flexible, electrically conductive hinge; (ii) electrically connecting the film with $\mathrm{Cu}$ tape, held in place with $\mathrm{NdFeB}$ magnets. This study implements the design investigated by Thompson using the second method of connecting the film together, as the method of masking allows the actuator to produce a clover-like shape under actuation, which impedes control and stability of the actuator. However, limitations arising through the use of $\mathrm{Cu}$ tape may include oxidation of the $\mathrm{Cu}$, converting the tape to an insulator, thus limiting the actuation of the PPy components connected by copper tape. For optimisation of the PPy film in this study, the geometry of PPy bending actuators is limited to $50 \mu \mathrm{m}$ thickness and $4 \mathrm{~mm}$ width as the charge distribution has been found to be no longer uniform at thicknesses greater than 50$60 \mu \mathrm{m}$ and curling is prominent at widths greater than $4 \mathrm{~mm}$, producing an increase in elastic stiffness of the actuator $[2,8]$. These limitations are also expected to be evident in PPy linear actuators.

A significant amount of work to determine the performance of bending PPy actuators has been previously conducted $[5,8]$ However, the complexity and greater degree of variability in a linear actuator means that the performance of a bending actuator may not directly translate to linear actuators. The modelling of linear actuators is necessary to accurately predict the force, displacement and work outputs for control of the actuator in practical applications. Modelling and optimisation work on PPy bending actuators by Alici, Metz \& Spinks [2], in which a mathematical model to calculate the expected bending behaviour of PPy bending actuators was developed, and suggests that as the length of the actuator decreases, force output increases. This study has investigated the effect of the length of PPy components in linear actuators to determine if length has a similar effect on outputs in linear actuators. Combinations of $2 \mathrm{~mm}, 3 \mathrm{~mm}, 4 \mathrm{~mm}$ wide and $10 \mathrm{~mm}, 15 \mathrm{~mm}$ and $20 \mathrm{~mm}$ long linear actuators, where the length refers to the length of each component, were experimentally evaluated for force and displacement outputs. It was found that an increase in the length of the PPy links in the linear actuators allowed a greater range of motion, whilst shorter and wider actuators lifted greater loads. The linear actuators were also compared to bending actuators of corresponding dimensions and it was found that the bending actuators had a greater range of motion and the linear actuators lifted heavier loads. As the proposed linear actuation mechanism converts the rotational work into the linear work, ideally they are equal to each other, it is expected that while the displacement is decreasing, the force is increasing, and vice versa. 


\section{Functioning Principle of Linear Actuation System}

As the polymer actuators considered in this study are cantilevered from one end, they generate a rotary (bending) type motion. This is analogous to a single jointed crank motion. A single degree of freedom mechanism such as a four-bar mechanism does not allow control the output motion. With this in mind, a five-bar mechanism which needs two inputs (two bending type polymer actuators) is one of the multi crank mechanisms having practical importance, especially for following any arbitrary trajectory precisely [9]. This mechanism serves as a bending to-linear motion converter; converting bending angle into a linear movement, and bending moments into linear forces.

\subsection{Kinematic Analysis}

The topology of the mechanism is depicted in Figure 1, where it is assumed that the mechanism links are rigid for the sake of generating a pseudo-rigid-body-model for the size and operation optimization of this linear motion mechanism. The joints connected to the frame are the active ones. The others are passive. For the joint inputs $\theta_{1}$ and $\theta_{2}$ and the specified link lengths $L_{0}, L_{1}, L_{2}, L_{3}, L_{4}$ the analytical expressions for the coordinates of the output point $\mathbf{P}$ are obtained using the following algorithm [9];

$$
\begin{gathered}
\overrightarrow{\mathrm{L}}_{1}=\mathrm{L}_{1} \cos \theta_{1} \overrightarrow{\mathrm{i}}+\mathrm{L}_{1} \sin \theta_{1} \overrightarrow{\mathrm{j}}, \\
\overrightarrow{\mathrm{L}}_{5}=\left(\mathrm{L}_{0}+\mathrm{L}_{4} \cos \theta_{2}\right) \overrightarrow{\mathrm{i}}+\mathrm{L}_{4} \sin \theta_{2} \overrightarrow{\mathrm{j}}, \\
|\mathrm{AB}|=\overrightarrow{\mathrm{R}}=\left|\mathrm{O}_{1} \mathrm{~B}\right|-\overrightarrow{\mathrm{L}}_{1}=\mathrm{C} \overrightarrow{\mathrm{i}}+\mathrm{D} \overrightarrow{\mathrm{j}}, \\
\mathrm{C}=\left|\mathrm{O}_{1} \mathrm{~B}\right|_{\mathrm{x}}-\mathrm{L}_{1 \mathrm{x}}, \quad \mathrm{D}=\left|\mathrm{O}_{1} \mathrm{~B}\right|_{\mathrm{y}}-\mathrm{L}_{1 \mathrm{y}}, \\
\mathrm{R}=\sqrt{\mathrm{C}^{2}+\mathrm{D}^{2}}, \\
\mathrm{Q}=\frac{\mathrm{L}_{2}^{2}+\mathrm{R}^{2}-\mathrm{L}_{3}^{2}}{2 \mathrm{R}^{2}},
\end{gathered}
$$

The coordinates $\left(\mathrm{x}_{\mathrm{P}}, \mathrm{y}_{\mathrm{P}}\right)$ of the output point $\mathrm{P}$ are;

$$
\begin{aligned}
& \mathrm{x}_{\mathrm{P}}=\mathrm{x}_{\mathrm{A}}+\mathrm{Q} \mathrm{C}-\mathrm{D} \sqrt{\frac{\mathrm{L}_{2}^{2}}{\mathrm{R}^{2}}-\mathrm{Q}^{2}}, \\
& \mathrm{y}_{\mathrm{P}}=\mathrm{y}_{\mathrm{A}}+\mathrm{Q} \mathrm{D}+\mathrm{C} \sqrt{\frac{\mathrm{L}_{2}^{2}}{\mathrm{R}^{2}}-\mathrm{Q}^{2}},
\end{aligned}
$$

Depending on the link lengths, the expression in the square-root can become negative, which suggest that some kinematic design constraints are not satisfied. For a linear motion output, the output point can be constrained to move in a rectilinear fashion. This requires that $\theta_{1}=\theta_{2}$ AND $L_{1}=L_{4}$ AND $L_{2}=L_{3}$. Such a mechanism 


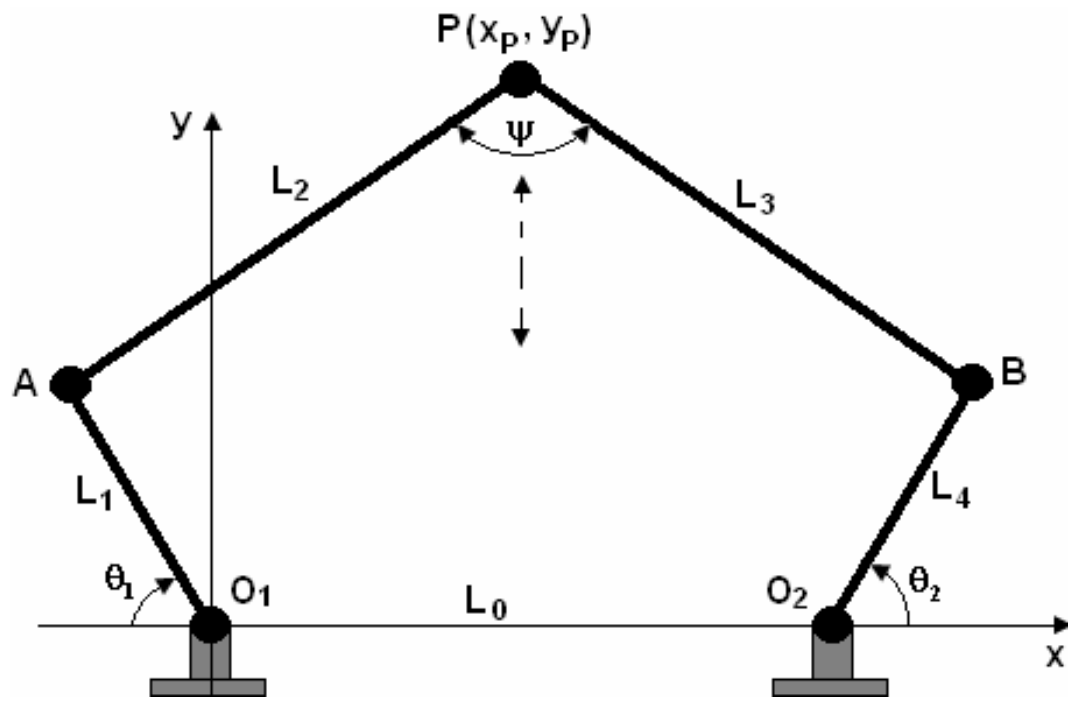

Fig. 1. Proposed linear actuation module based on a parallel five-bar mechanism

will convert all of its rotary work into the linear work - an efficient motion converter. The analytical expressions for the output point of such a linear motion module are obtained as

$$
\begin{aligned}
& \mathrm{x}_{\mathrm{P}}=-\mathrm{L}_{1} \cos \theta_{1}+\frac{1}{2}\left[\mathrm{~L}_{0}+\mathrm{L}_{1}\left(\cos \theta_{2}+\cos \theta_{1}\right)\right] \\
& -\mathrm{L}_{1}\left(\sin \theta_{2}-\sin \theta_{1}\right) \sqrt{\frac{\mathrm{L}_{2}^{2}}{\mathrm{R}^{2}}-\frac{1}{4}} \\
& \mathrm{y}_{\mathrm{P}}=\mathrm{L}_{1} \sin \theta_{1}+\frac{\mathrm{L}_{1}}{2}\left(\sin \theta_{2}-\sin \theta_{1}\right) \\
& +\left[\mathrm{L}_{0}+\mathrm{L}_{1}\left(\cos \theta_{2}+\cos \theta_{1}\right)\right] \sqrt{\frac{\mathrm{L}_{2}^{2}}{\mathrm{R}^{2}}-\frac{1}{4}}
\end{aligned}
$$

where

$$
\begin{aligned}
& \mathrm{R}^{2}=\mathrm{L}_{0}^{2}+2 \mathrm{~L}_{0} \mathrm{~L}_{1}\left(\cos \theta_{2}+\cos \theta_{1}\right) \\
& +2 \mathrm{~L}_{1}^{2}\left[1+\cos \left(\theta_{1}+\theta_{2}\right)\right]
\end{aligned}
$$

Depending on the link length of the mechanism, Eq.6 simplifies to

$$
\begin{aligned}
& \text { i) For } L_{1}=L_{2} \text { and } \theta_{1}=\theta_{2} \Rightarrow x_{P}=\frac{L_{0}}{2} \text { and } \\
& \mathrm{y}_{\mathrm{P}}=\mathrm{L}_{1} \sin \theta_{1} \\
& +\frac{1}{2} \sqrt{4 \mathrm{~L}_{1}^{2}-\mathrm{L}_{0}^{2}-4 \mathrm{~L}_{0} \mathrm{~L}_{1} \cos \theta_{1}-4 \mathrm{~L}_{1}^{2} \cos ^{2} \theta_{1}}
\end{aligned}
$$


ii) For $\mathrm{L}_{0}=0$ and $\theta_{1}=\theta_{2} \Rightarrow \mathrm{x}_{\mathrm{P}}=0 \quad$ and

$$
\mathrm{y}_{\mathrm{P}}=\mathrm{L}_{1} \sin \theta_{1}+\sqrt{\mathrm{L}_{2}^{2}-\mathrm{L}_{1}^{2} \cos ^{2} \theta_{1}}
$$

iii) For $\mathrm{L}_{0}=0, \mathrm{~L}_{1}=\mathrm{L}_{2}$ and $\theta_{1}=\theta_{2} \Rightarrow \mathrm{x}_{\mathrm{P}}=0$ and $\mathrm{y}_{\mathrm{P}}=2 \mathrm{~L}_{1} \sin \theta_{1}$

When choosing the link sizes, it is important for effective force transmission to minimize the variation of the transmission angle $\psi$ from $90^{\circ}$; the acceptable range is $90^{\circ} \mp 40^{\circ}$ [10]. It is mathematically expressed as

$$
\cos \psi=1-\frac{1}{2}\left(\frac{\mathrm{R}}{\mathrm{L}_{2}}\right)^{2}
$$

For $50^{\circ} \leq \psi \leq 130^{\circ}, 0.845 \leq\left(\frac{\mathrm{R}}{\mathrm{L}_{2}}\right) \leq 1.8126$. With reference to Eq.8, for a given $\mathrm{L}_{0}$ and $\mathrm{L}_{1}$, the range of $\mathrm{L}_{2}$ satisfying the effective transmission angles can be calculated. It must be noted that $\mathrm{L}_{0}$ should be greater than $\mathrm{L}_{1}$ to prevent any physical interference among the mechanism links. A practical ratios of $1.5 \leq\left(\frac{\mathrm{L}_{0}}{\mathrm{~L}_{1}}\right) \leq 3.0$ and $1.0 \leq\left(\frac{\mathrm{L}_{2}}{\mathrm{~L}_{1}}\right) \leq 2.0$ should result in proportionate link lengths and transmission angles. For the data shown in Table 1, the transmission angle and the vertical movement of the linear actuation module are calculated and presented in Figure 2.

Table 1. Numerical values of the parameters for the exemplary results shown in Figure 2

\begin{tabular}{|c|}
\hline Parameters \\
\hline $\mathrm{L}_{0}=40 \mathrm{~mm}$ \\
\hline $\mathrm{L}_{1}=\mathrm{L}_{3}=20 \mathrm{~mm}$ \\
\hline $\mathrm{L}_{2}=\mathrm{L}_{4}=22 \mathrm{~mm}$ \\
\hline$\theta_{1}=\theta_{2}=90^{\circ}$ to $115^{\circ}$ \\
\hline
\end{tabular}

The corresponding generalized relationship between rate of change $\dot{\Theta}=\left[\begin{array}{ll}\dot{\theta}_{1} & \dot{\theta}_{2}\end{array}\right]^{T}$ of the input bending angles and the output velocity vector $\dot{X}=\left[\begin{array}{ll}\dot{x}_{P} & \dot{y}_{P}\end{array}\right]^{T}$ is given by [11].

$$
\dot{\mathrm{X}}=\left[\frac{\partial \mathrm{x}_{\mathrm{P}}}{\partial\left(\theta_{1}, \theta_{2}\right)} \frac{\partial \mathrm{y}_{\mathrm{P}}}{\partial\left(\theta_{1}, \theta_{2}\right)}\right]^{\mathrm{T}} \dot{\Theta}=\mathrm{J} \dot{\Theta}
$$

where $\mathrm{J}$ is the mechanism Jacobian matrix. 


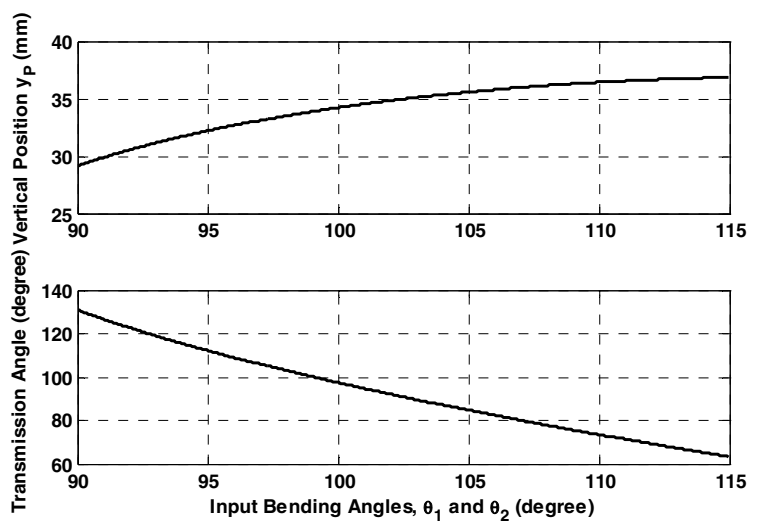

Fig. 2. Variation of the vertical position of the output point $P$ and the transmission angle with the input bending angles. The net vertical distance is $7.6858 \mathrm{~mm}$.

\subsection{Force Analysis}

Assuming that two active links $\mathrm{O}_{1} \mathrm{~A}$ and $\mathrm{O}_{2} \mathrm{~B}$ made of electroactive polymers generate bending moments $\mathrm{M}_{1}$ and $\mathrm{M}_{2}$, which act on the actuation module in the opposite directions. Assume that the output point $\mathrm{P}$ can apply a planar force vector of $F_{p}=F_{x} \vec{i}+F_{y} \vec{j}$ to the environment to realise a functional task. Please recall that the mechanism converts the work in the bending coordinates $\Theta$ into the work in linear coordinates $\mathrm{X}$. These two works are ideally equal to each other.

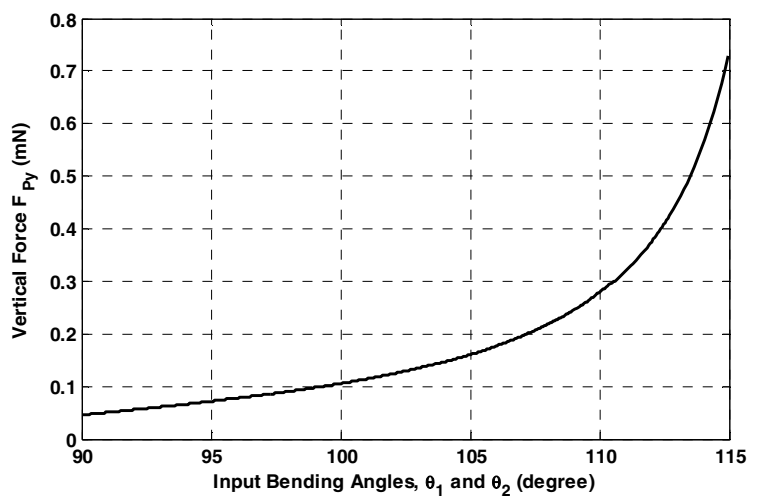

Fig. 3. Variation of the vertical force component of the output point $\mathrm{P}$ with the input bending angles. The net vertical force is $0.6837 \mathrm{mN}$. The horizontal component of the force is zero.

Using the duality between the generalized relationships for motion and force transfer between the actuation and output spaces, the following force relationship is obtained [11]; 


$$
\mathrm{M}=\mathrm{J}^{\mathrm{T}} \mathrm{F}_{\mathrm{P}} \Rightarrow \mathrm{F}_{\mathrm{P}}=\mathrm{J}^{-\mathrm{T}} \mathrm{M}
$$

where $\mathrm{F}_{\mathrm{P}}=\left[\begin{array}{ll}\mathrm{F}_{\mathrm{x}} & \mathrm{F}_{\mathrm{y}}\end{array}\right]^{\mathrm{T}}$ and $\mathrm{M}=\left[\begin{array}{ll}\mathrm{M}_{1} & \mathrm{M}_{2}\end{array}\right]^{\mathrm{T}}$ and $(.)^{\mathrm{T}}$ denotes transposition. For the data in Table 1 and $M=\left[\begin{array}{ll}1 & 1\end{array}\right]^{\mathrm{T}} \mathrm{Nmm}$, the force output at point $\mathrm{P}$ is calculated using Eq.10 and is presented in Figure 3. Because the mechanism link lengths are chosen such that it can generate a vertical movement, the mechanism will not create any horizontal force component. These kinematic and force analyses suggest that it is possible to optimize the topology of the mechanism for an efficient motion and force transmission.

\section{Conducting Polymer Actuators: Synthesis and Actuation Principle}

The PPy film was manufactured by combining a solution of $0.1 \mathrm{M}$ pyrrole monomer with $0.1 \mathrm{M}$ lithium triflouromethanesulfonimide $\left(\mathrm{Li}^{+} \mathrm{TFSI}{ }^{-}\right.$) in propylene carbonate (PC) with $1 \% \mathrm{H}_{2} \mathrm{O}$, degassed with nitrogen gas and stirred for 15 minutes. This solution was used to grow the PPy onto a gold sputter coated, $0.45 \mu \mathrm{m}$ Millipore Immobilon-P porous poly(vinylidene fluoride) (PVDF) film by electrodeposition over a period of 12 hours at $-33^{\circ} \mathrm{C}$ with a current density of $0.1 \mathrm{~mA} \cdot \mathrm{m}^{-2}$. After polymerization, the film was washed in acetone and then resoaked in a $0.1 \mathrm{M}$ LiTFSI electrolyte and then stored away from oxygen. Actuators were then cut to desired size using a scalpel and were limited to size by the area of the $40 \mathrm{~mm} \times 55 \mathrm{~mm}$ film. The resulting film consists of two PPy outer layers and two gold layers separated by a PVDF core is shown in Figure 4.

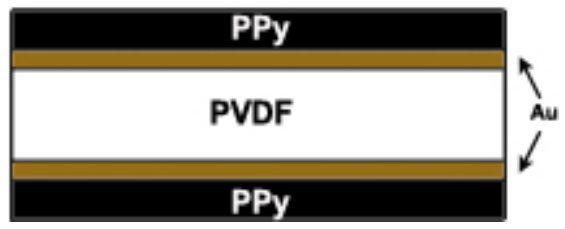

Fig. 4. General structure of trilayer polymer actuator

After doping with electrolyte, both the PPy layers of a bending actuator become partially oxidised (Figure 5a) and upon application of a positive voltage, one of the polymer layers is highly oxidized and the other is reduced. In an attempt to neutralise the charge imbalance, ions transfer from the electrolyte into the polymer layers. In LiTFSI doped actuators, TFSI ${ }^{-}$anions move into interstitial spaces in the polymer backbone of the oxidized layer, causing it to expand. Simultaneously, the opposing polymer film is reduced and contracts by removing TFSI $^{-}$ions, overall creating a bending motion (Figure 5b.) By applying a square wave voltage, the actuator is allowed to return it to its original position and continue to bend through the neutral position so that the actuator may bend alternately in both directions. 

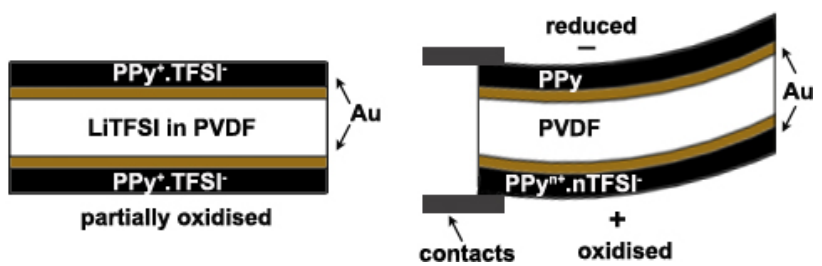

Fig. 5. Schematic of PPy actuator doped in LiTFSI (a) partially oxidised (b) under an applied voltage

To produce the linear actuators, two pieces of PPy film cut to equal dimensions were connected together in parallel at their tips with copper tape and two $\varnothing 3 \mathrm{~mm} \times$ $0.5 \mathrm{~mm}$ neodymium iron boron $(\mathrm{NdFeB})$ magnets one either side of each tip (Figures 6-7.) Once placed between the electrodes, the PPy configuration effectively produces four PPy components, with the length referred to throughout this paper as the length depicted in Figure $7 \mathrm{~b}$.

Under an applied voltage, it was observed that the PPy linear actuator produced the shape as shown in Figure 8. In this case, the outer PPy layer of the upper components is oxidised and the inside layer is reduced, and under no constraints, almost uniform curvature may be experienced. The lower components may be activated; however the possible oxidation of the $\mathrm{Cu}$ tape suggests that the lower components remain either inactivated. This linear design restricts the bending motion of the actuators due to the presence of the magnets used to clamp the copper tape to the actuators (Figure 8). For the shorter lengths, a greater percentage of the actuator is affected by the clamping induced by the magnets and as a result, the actuator has a more elongated appearance (Figure 9). Due to this elongation, it doesn't move as far in the vertical direction as it potentially could. To overcome this limitation, another method of electrically connecting the upper and lower actuator components that does not restrict the motion is necessary. For this to be possible, the electrical connection must be flexible and able to bond to the actuator without the assistance of magnets. Although the magnets restrict motion, they do provide some stability which is important in attempting to control the actuator.

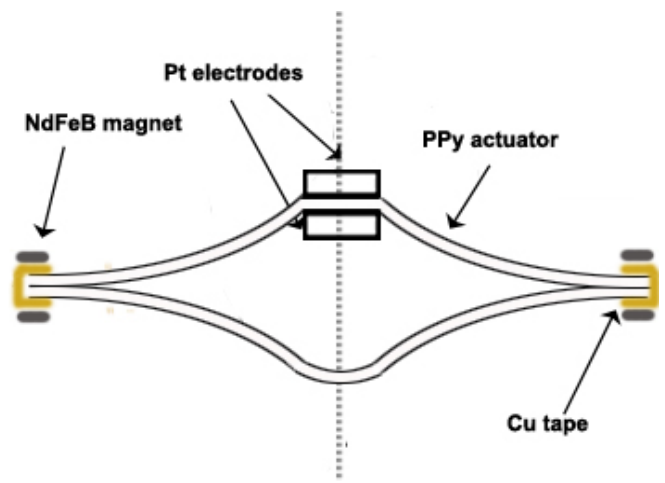

Fig. 6. Configuration of the linear PPy actuator with $\mathrm{Cu}$ tape and $\mathrm{NdFeB}$ magnets 


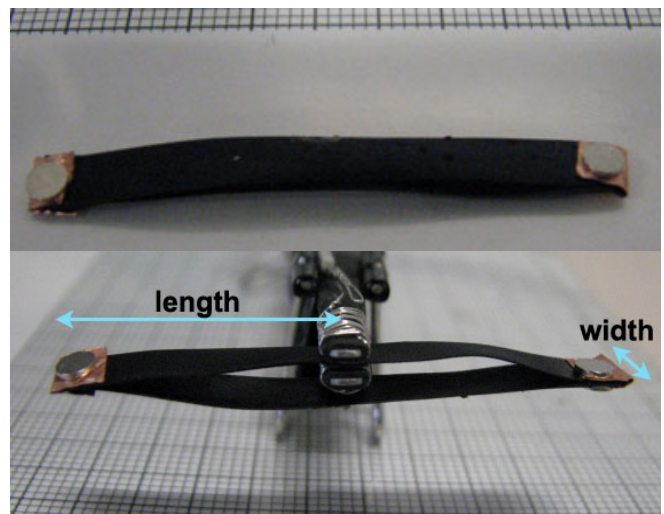

Fig. 7. Linear PPy actuator in neutral position

\section{Performance Characterization Results}

Linear actuators shown in Figures 6-7 with link lengths of 10mm, 15mm, 20mm and widths of $2 \mathrm{~mm}, 3 \mathrm{~mm}, 4 \mathrm{~mm}$ were manufactured and tested under a series of applied loads. Loads were added to the bottom tip of the actuators and the overall displacement of the base tip was measured visually using a grid paper, as illustrated in Figure 10a. The initial load used was the non-magnetic wire hook and a $0.266 \mathrm{~g}$ $\mathrm{NdFeB}$ magnet glued to its base (Figure 10b), with incremental loads applied by adding magnets of either $0.266 \mathrm{~g}$ or $0.54 \mathrm{~g}$ to the base of the wire hook. Voltage was maintained at $+/-1 \mathrm{~V}$ to avoid over-oxidation and reduce the variation in results that may arise due to early onset of fatigue. The frequency of the square voltage inputs was held constant for all experiments at $0.5 \mathrm{~Hz}$ to allow sufficient response time. Typical voltage, current and charge data recorded by the datalogging system used is presented in Figure 11.

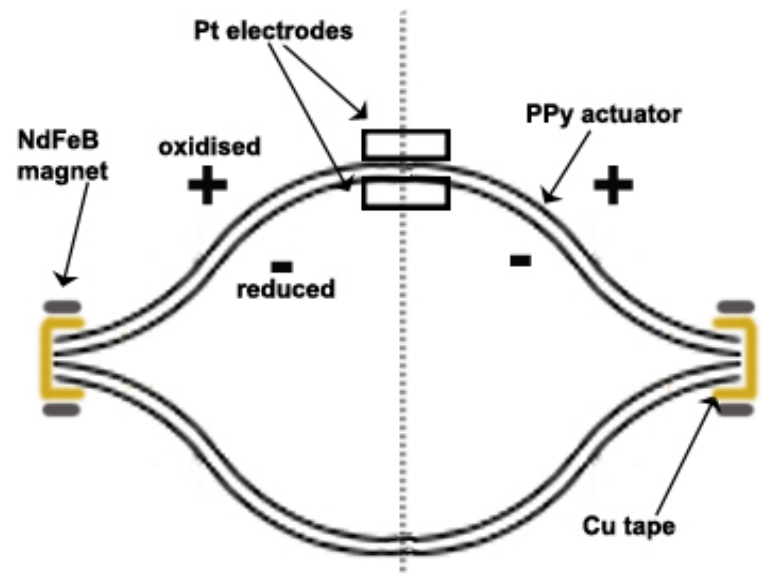

Fig. 8. Shape of actuator under actuation 


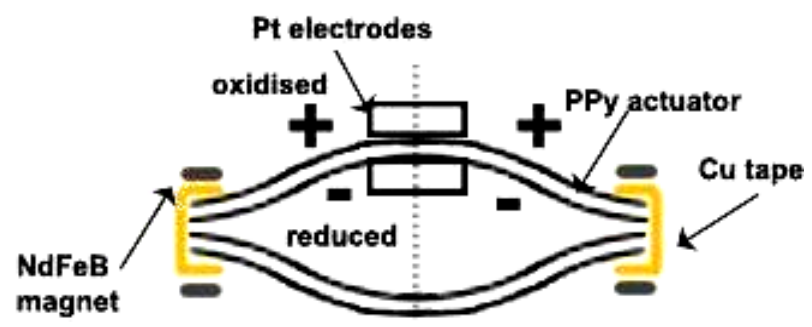

Fig. 9. Shape of shorter actuators under actuation

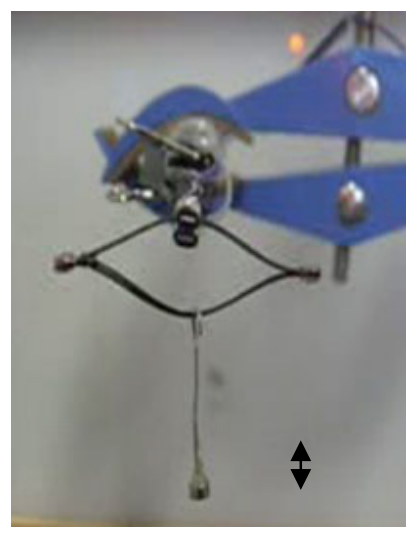

(a)

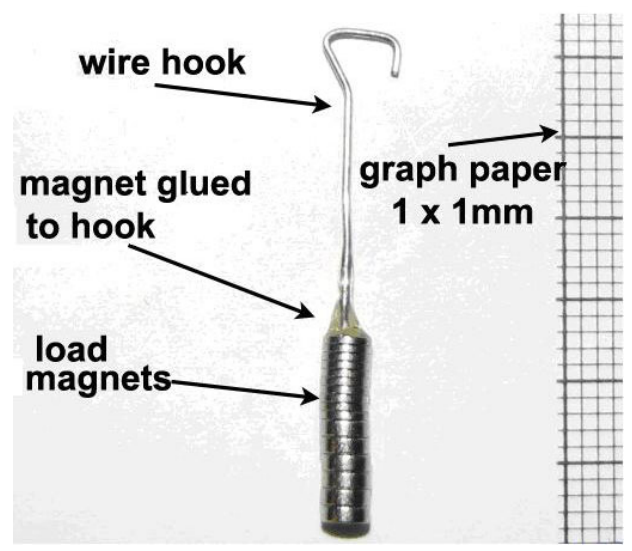

(b)

Fig. 10. (a) PPy linear actuator with a load. (b) Wire hook; used to separate magnet loads from magnets on the actuator.

Figures 12(a)-(i) provide an overview of the base tip position in extended and contracted positions under given applied loads, with the electrode contacts as the reference point. In all actuators, as the load increases the position of the base tip below the reference point also increases. Generally, as the width decreases the position below the electrode increases and is more evident in the $15 \mathrm{~mm}$ long actuators. As width increases, the actuator becomes stiffer which allows a smaller deflection below the reference point under a load than an actuator of a small width.

Displacement increases and then decreases for increasing load as shown in Figure 12(d)-(i) for the $15 \mathrm{~mm}$ and $10 \mathrm{~mm}$ long actuators. This behaviour is because under no load, the actuators are restricted in their movement by the magnets clamping the $\mathrm{Cu}$ tape in place. The clamping effect has a greater impact on shorter actuators because, as a percentage, a greater proportion of the actuator is restricted than longer actuators. Under applied loads, the shorter actuators are able to move because the load forces the actuator base tip to be displaced to lower than the original position, and under an applied voltage the actuator attempts to return to the original position and produces an upward motion. For this reason, the shorter actuators may only be suitable for applications requiring a pulling load, and if shorter actuators are required for pushing, then another method of connecting the actuators that provides less restriction on motion is required. 


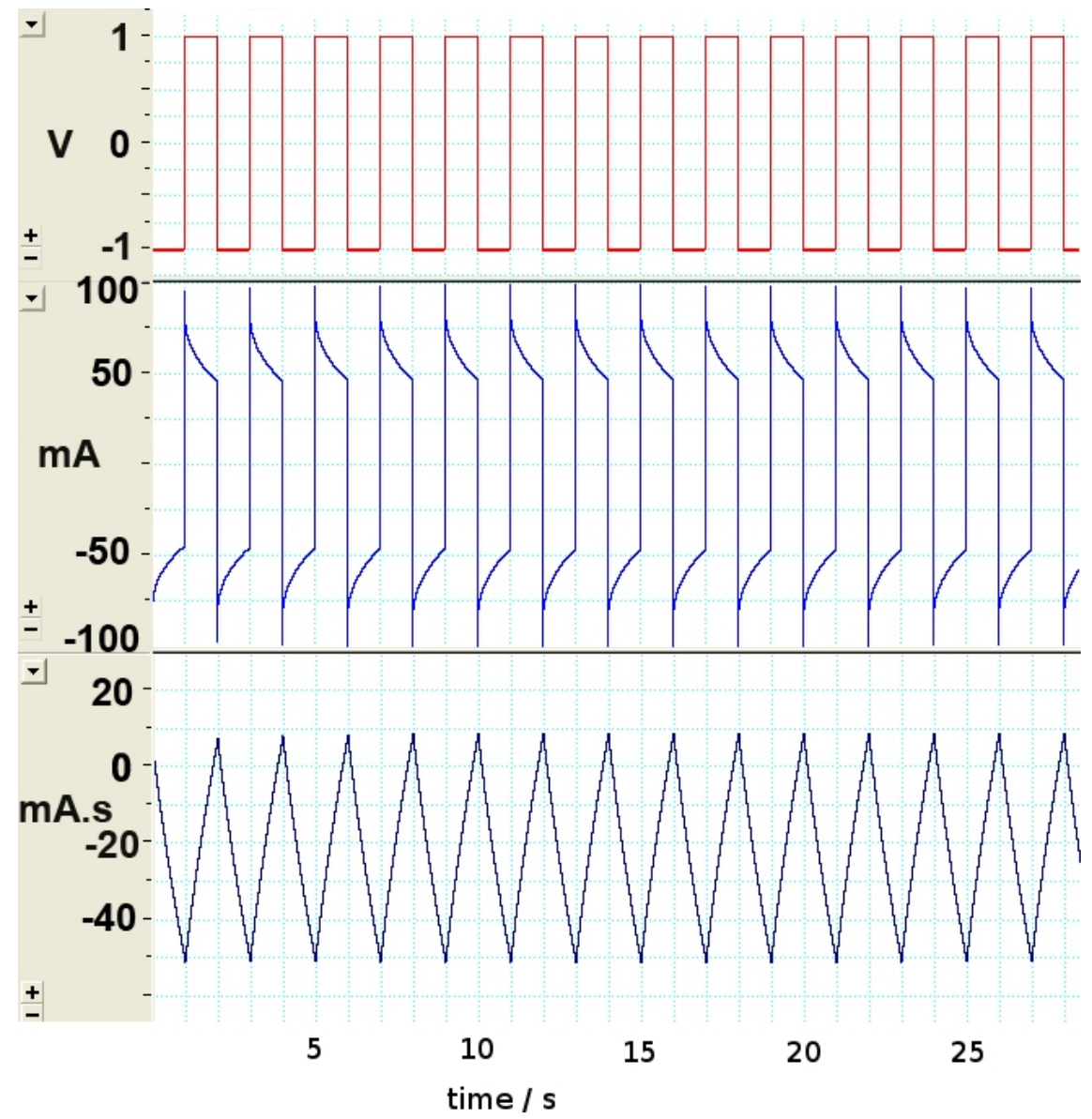

Fig. 11. Example voltage, current and charge input data for $20 \mathrm{~mm} \times 2 \mathrm{~mm}$ linear PPy actuator at $0.5 \mathrm{~Hz}$
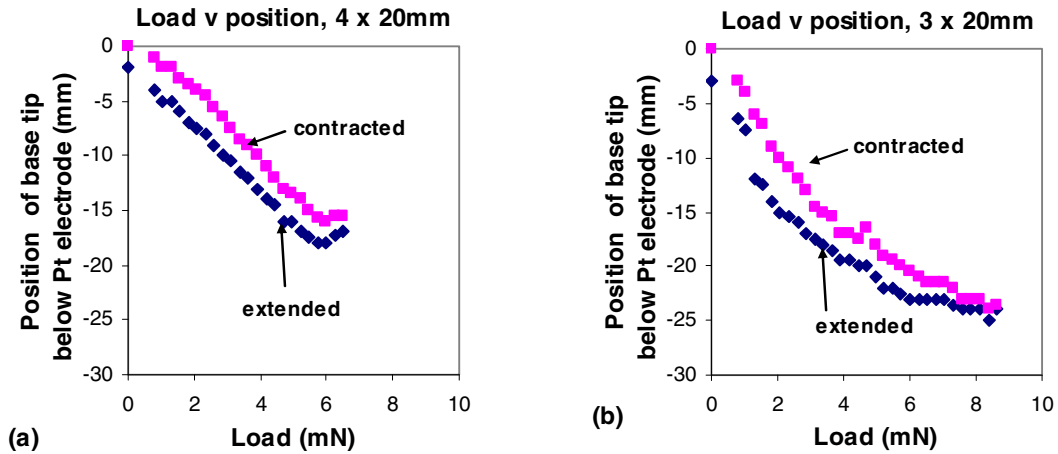

Fig. 12. Position of base tip of linear actuators under applied load 

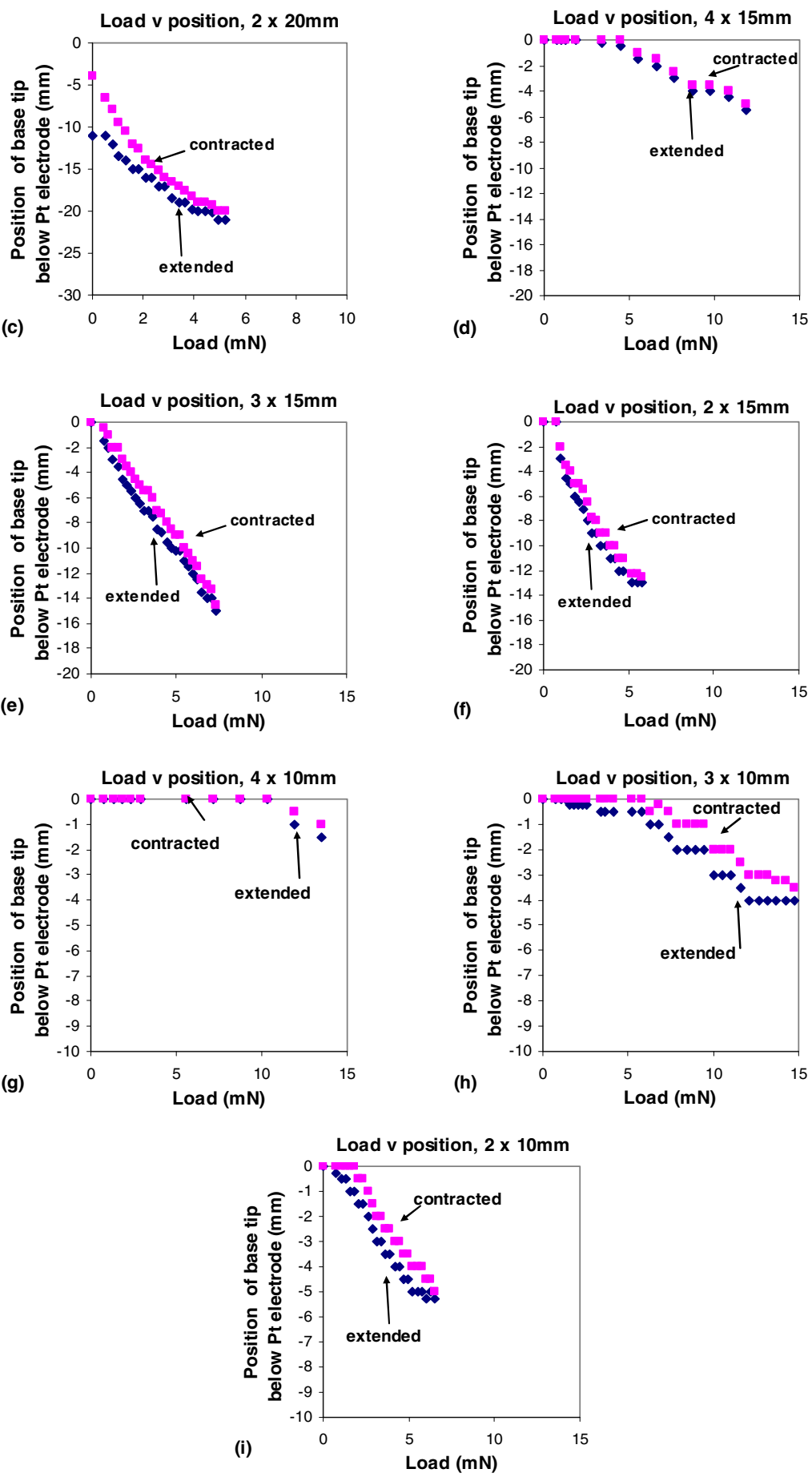

Fig. 12. (continued) 

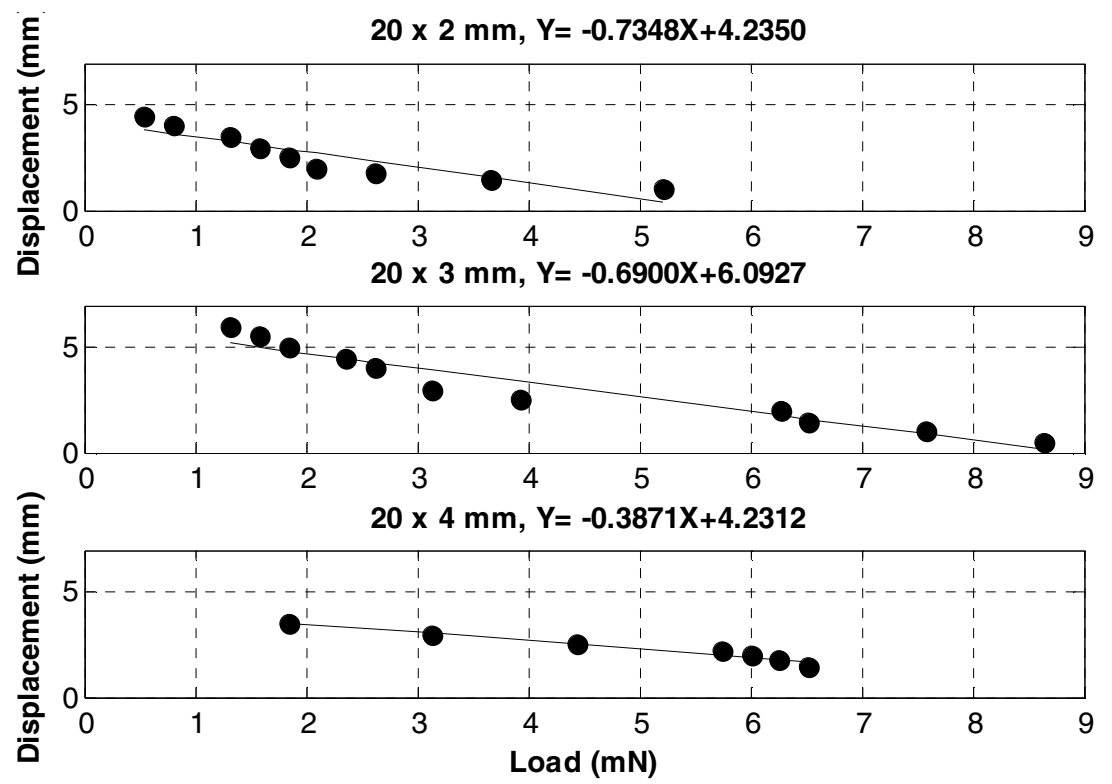

Fig. 13. Variation of the tip displacement of the linear actuation module with the payload

A comparison of load and displacement output between the linear and cantilever actuators of corresponding length has revealed that the cantilever actuators provide a greater range of motion in the vertical direction, while the linear actuator lifted heavier loads. The linear actuators are effectively 4 times the volume of their corresponding bending actuator. However, they produced much less displacement due to the restriction in motion provided by the magnet connections. The cantilever actuators have the ability to move above and below the electrodes whereas the linear actuators are limited to motion below the electrodes only. Although much less displacement is achieved, the benefit of a linear actuator is that motion in one direction is more controlled than the motion of a bending actuator. For displacement output, the linear actuators with 20mm PPy links produced the greatest displacement than actuators with shorter PPy links over a range of loads. Figure 13 depicts the displacement in the vertical direction for linear actuators of varied width with $20 \mathrm{~mm}$ length. As width of the PPy links increases, a greater displacement output for a given load may be expected. However, as width increases, the PPy film has a tendency to curl across the width, particularly in actuators for $4 \mathrm{~mm}$ wide or greater [4]. Curling increases stiffness and thus decreases the potential displacement output, hence the $4 \mathrm{~mm}$ wide actuator produced a slightly smaller displacement than actuator with $3 \mathrm{~mm}$ wide PPy links.

The calculated work output for actuators of varying length and width is presented in Fig. 14 and compared by the width for each actuator length. For the $20 \mathrm{~mm}$ long 
actuators, the $3 \mathrm{~mm}$ and $4 \mathrm{~mm}$ wide actuators produced a similar work output, and were significantly higher than the $2 \mathrm{~mm}$ wide actuator. As width increases, the actuators should experience more charge and therefore should produce greater work. However, the $4 \mathrm{~mm}$ wide actuator may have experienced curling, in which it would have contributed some of its energy to curling across the width of the actuator. The curling effect may also impede motion by increasing the stiffness of the actuator, therefore reducing work output by restricting motion.

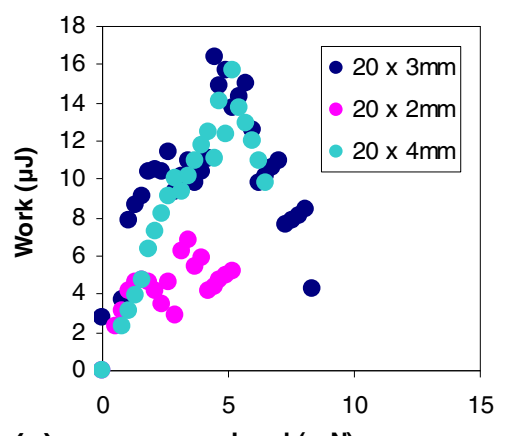

(a)

$$
\text { Load }(\mathrm{m} \mathrm{N})
$$
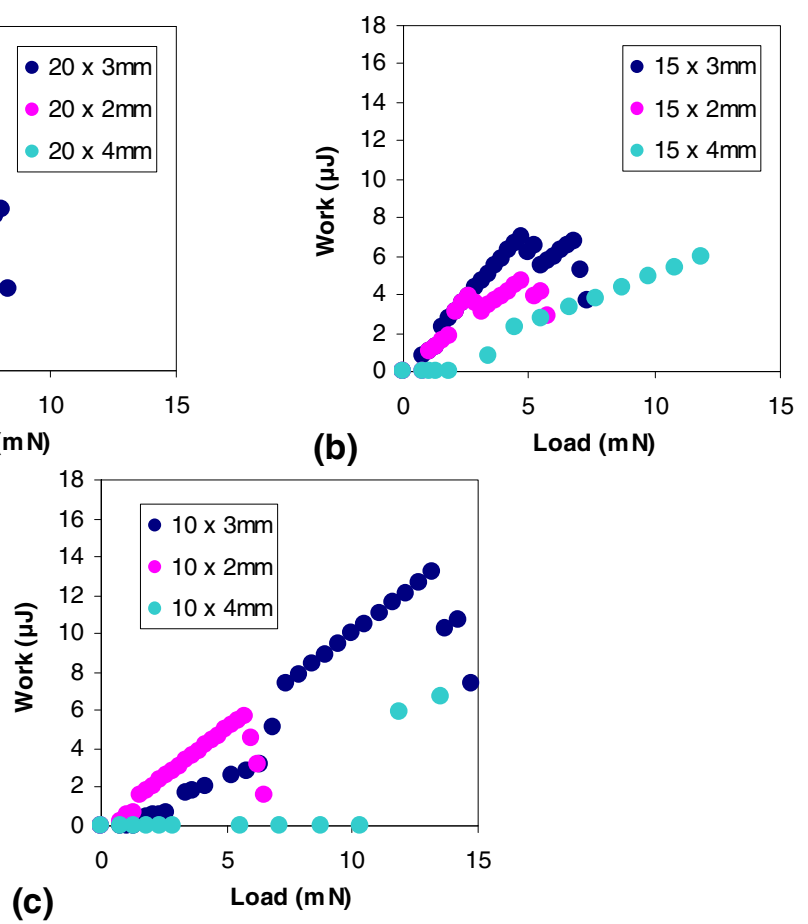

Fig. 14. Work versus load for PPy linear actuator: (a) $20 \mathrm{~mm}$ long components (b) $15 \mathrm{~mm}$ long components (c) $10 \mathrm{~mm}$ long components

Work versus load plots should depict a parabola shape, which is evident in the $20 \mathrm{~mm}$ long actuators (Fig.14a) The parabola shape is not present in the work versus load plots for the $15 \mathrm{~mm}$ and $10 \mathrm{~mm}$ long actuators (Fig. 14b-c) because a significant proportion of work being done was being opposed by clamping of the magnets at the hinges. Therefore, the magnets impeded the work output for shorter actuators, and the development of an alternate method of joining the components may allow greater output for shorter actuators. Maximum work for the $4 \mathrm{~mm} \times 15 \mathrm{~mm}$ and $4 \mathrm{~mm} \times$ $10 \mathrm{~mm}$ actuators is not depicted in the Fig. 14b-c, however at the maximum work measured, these actuators produced very little displacement (Fig 13.) These results 
suggest that the increased stiffness of the actuator created by the curling effect of the actuator could be useful in applications where higher loads and very small displacements are required.

Work density can be used to compare actuators of different geometries, performance and efficiency and is also a good measure to determine the actuators that are not performing near their potential. Fig. 15 compares the work density versus load of the $20 \mathrm{~mm}$ long actuators with varying width. For these configurations, the $3 \mathrm{~mm}$ wide actuator shows a significantly higher work density than both the $2 \mathrm{~mm}$ and $4 \mathrm{~mm}$ wide actuators. The $2 \mathrm{~mm}$ and $4 \mathrm{~mm}$ actuators exhibited similar work densities, and further testing would be required to determine which is more efficient. The $2 \mathrm{~mm}$ actuator has a similar density to the $3 \mathrm{~mm}$ wide actuator at lower loads $(<1 \mathrm{mN})$, but has significantly lower work density at higher loads. The $4 \mathrm{~mm}$ actuator is less efficient than the $3 \mathrm{~mm}$ actuator, due to a proportion of the input energy being contributed to curling across its width. Because of its higher work density, the $3 \mathrm{~mm}$ wide actuator would be more preferable when constraints such as cost and volume exist. However, because the $2 \mathrm{~mm}$ and $4 \mathrm{~mm}$ wide actuators have a lower work density than the $3 \mathrm{~mm}$ wide actuator, there is greater potential for their work output to be improved. If the efficiency of the $4 \mathrm{~mm}$ wide actuator was improved then it would have a higher work output than the $3 \mathrm{~mm}$, which is shown in Figure 14.

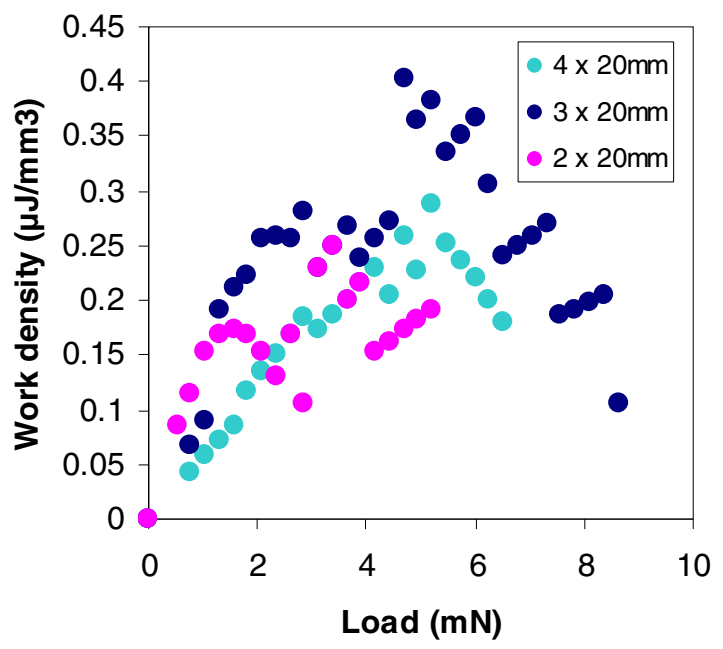

Fig. 15. Work density of PPy linear actuator with $20 \mathrm{~mm}$ long components

\section{Conclusions}

A linear actuator system based on the bending motion of conducting polymer actuators operating in air is presented, including an analytical model to estimate the linear movement and the force output of the mechanism. The mechanism is basically a motion and force transmission system, converting the bending work provided by the electroactive polymer actuators into Cartesian work. The experimental results 
presented demonstrate that the conducting polymer actuators generate enough displacement and force to handle a range of practical payloads. Another outcome of this study is that when the bending type- low power consuming polymer actuators are tailored properly, they can be used to generate a rectilinear motion with enough force output.

Future work involves deriving a more accurate analytical model taking into account the deflections of the mechanism links and verifying the model experimentally. Improvements may also be made to the hinge connections of the linear actuator, by replacing the copper connections with an inert, conductive material such as gold or platinum.

Acknowledgements. The authors thank Dr Stephen W. John for his help in synthesizing the bulk actuator sheet and construction of the actuation module. This work was supported in part by the ARC Centre of Excellence for Electromaterials Science (CE0561616).

\section{References}

1. Thompson, F.: Hybrid linear actuator based on conducting polymers. Faculty of Engineering. University of Wollongong (2007)

2. Alici, G., Metz, P., Spinks, G.: A mathematical model to describe bending mechanics of Polypyrrole (PPy) Actuators. In: Proceedings of the 2005 IEEE/ASME International Conference on Advanced Intelligent Mechatronics, Monterey, California (2005)

3. EAMEX Corporation, http: / /www. eamex.co.jp/index_e.html

4. Alici, G., Spinks, G., Huynh, N., Sarmadi, L., Minato, R.: Establishment of a Biomimetic Device Based on Tri-layer Polymer Actuators - Propulsion Fins. Bioinspr. Biomim. 2, 18 30 (2007)

5. Alici, G., Huynh, N.: Performance Quantification of Conducting Polymer Actuators for Real Applications: A Microgripping System. ISEE-ASME T Mech. 12, 73-84 (2007)

6. Otero, T., Cortes, M., Vazquez Arenas, G.: Linear movements from two bending triplelayers. Electrochim Acta 53, 1252-1258 (2007)

7. Yamakita, M., Kamamichi, N., Yashuaki, K., Aska, K., Luo, A.: Development of an artificial muscle linear actuator using ionic polymer metal composites. Adv. Robotics 13, 383-399 (2004)

8. Metz, P., Alici, G., Spinks, G.: A finite element model for bending behaviour of conducting polymer electromechanical actuators. Sensor Actuat A-Phys., 130-131, 1-11 (2006)

9. Alici, G.: Determination of Singularity Contours for Five-bar Planar Parallel Manipulators. Robotica 18, 569-575 (2000)

10. Alici, G., Shirinzadeh, B.: Optimum synthesis of parallel manipulators based on kinematic isotropy and force balancing. Robotica 22, 97-108 (2004)

11. Alici, G., Shirinzadeh, B.: Enhanced Stiffness Modelling, Identification and Characterisation for Robot Manipulators. IEEE T. Robot. 21, 554-564 (2005)

12. John, S., Alici, G., Cook, C.: Validation of a Resonant Frequency Model for Polypyrrole Trilayer Actuators. ISEE-ASME T. Mech. 13, 401-409 (2008) 